\title{
Two models for psychophysical judgment: Scale invariance with changes in stimulus range
}

\author{
JOHN R. FREDERIKSEN \\ Brandeis University, Waltham, Massachusetts 02154
}

\begin{abstract}
Two scaling models are investigated, each of which includes parameters which reflect variation in the use of judgmental categories. In one model, response categories are represented as successive intervals on an underlying sensory continuum. In the other, they are represented as points. Psychophysical scales based upon either of these models are invariant up to a linear transformation when the range of stimulus magnitudes is manipulated. Successive intervals scale values obtained from magnitude estimates are also linearly invariant with changes in stimulus range. Similarities in the proximity functions for the two judgment models are examined.
\end{abstract}

The variations in psychophysical scales which occur when changes are made in experimental technique (Poulton, 1968) or in the subject's mode of responding (Stevens, 1960) have led investigators to seek psychophysical scaling models that give explicit recognition to the processes of judgment which take place in the scaling experiment (Baird, 1970; Braida \& Durlach, 1972; Durlach \& Braida, 1969; Gravetter \& Lockhead, 1973). For example, the range of stimulus magnitudes employed in a psychophysical scaling experiment has been shown to affect the value of the exponent in the psychophysical power function which is obtained when subjects make direct estimates of perceived stimulus magnitude (Poulton, 1968; Teghtsoonian, 1971). Increasing the stimulus range results in a decrese in the size of this exponent. The same variable affects the degree of stimulus confusion in an absolute identification task; two stimuli are more often confused when presented in the context of a wide range of stimulus values than when a narrow range is used (Hartman, 1954; Pollack, 1953). Variations in the mode of judgment can also lead to psychophysical scales which are curvilinearly related. In particular, scales based upon category ratings are logarithmically related to scales based upon quantitative estimates of sensory magnitude (Eisler, 1962; Galanter \& Messick, 1961; Stevens \& Galanter, 1957) when prothetic continua are being judged (Stevens, 1957). An explanation for the logarithmic relation between scales based upon discriminability and magnitude estimation scales begins with the observation that the variability in sensory impressions resulting from a stimulus is proportional to stimulus magnitude (Weber's law) (Eisler, 1962; Stevens, 1957); scales constructed on the basis of equalizing

Support for this study was received from NIH Institutional Grant No. 7044-06 to Brandeis University. Parts of this study were reported at the annual meetings of the Eastern Psychological Association on April 29, 1972. Requests for reprints should be addressed to John R. Frederiksen, Department of Psychology, Brandeis University, Waltham, Massachusetts 02154. units of variability (discriminability) at all points on the scale (Helm, Messick, \& Tucker, 1961) are then logarithmically related to scales in which variability increases (and discriminability decreases) with increases in stimulus scale value (Stevens, 1957, 1960). Differing principles of judgment are involved in magnitude estimation and category rating, and are presumably responsible for the differing psychophysical functions. Judgmental factors have also been implicated in explaining the stimulus range effect (Poulton, 1968), and there has been a growing interest in developing formal models for psychophysical judgment to explain such effects. Models for psychophysical judgment have been developed along the lines of choice theory (Luce \& Mo, 1965) and Thurstone's model for categorical judgment (Durlach \& Braida, 1969; Gravetter \& Lockhead, 1973). Durlach and Braida, for example, explain the stimulus range effect by assuming that stimuli are coded according to their relations with the general context represented by the stimulus set used in the experiment; this context-coding is assumed to become less reliable (more noisy) as the stimulus range is increased.

In this paper, two models for psychophysical judgment will be outlined and then evaluated with respect to the invariance of the resulting psychophysical scales, as changes are made in the range of stimulus magnitudes employed and the type of judgment demanded of the subject (quantitative or categorical). The scaling models examined here are judgment models in that they include parameters to represent the judgmental categories employed by the subject. These judgment parameters are, in theory, independently manipulable, just as the criterion $\beta$ in signal detection theory can be altered by changes in payoffs or subjective stimulus probabilities (Green \& Swets. 1966). In the first model to be examined here, Thurstone's successive intervals model (Saffir, 1937; Torgerson, 1958). judgmental categories are represented as a series of successive, nonoverlapping intervals on the latent sensory continuum. This 
model. as Green and Swets (1966) have pointed out, can be regarded as an extension of the theory of signal detectability. The judgment parameters are the boundaries separating adjacent category intervals on the sensory axis. In the second model, an extension of Coomb's unidimensional unfolding technique (Coombs. 1964. Chap. 5), judgment categories are represented in the same manner as stimuli-as points on the sensory continuum. The judgment parameters here might be thought of as representing a set of internal, idealized stimuli which serve as standards against which test stimuli are to be judged. By further specifying the form of the function relating observed response probabilities to distances between stimuli and response categories on the sensory scale, an interval scale can be constructed. While the formal assumptions of the two models are somewhat different, each judgment model provides a set of parameters representing judgment categories, which are distinct from other parameters, including the stimulus scale values. Variations in the range of stimulus magnitudes or in mode of response should influence only the judgmental parameters, leaving the psychophysical scale invariant up to a linear transformation. A final purpose of the present study is to examine the extent to which a single principle of precision (or, alternatively, of uncertainty) in judgment can account for stimulus range effects on psychophysical scales based upon both quantitative and categorical modes of judgment. To this end, a set of processing models for magnitude estimation will be examined which attempts to account for the stimulus range effects, and a model which explains the range effects in terms of precision or uncertainty in judgment will be shown to account for the effects of stimulus range on power law exponents.

\section{THE JUDGMENT MODELS}

The basic data in any psychophysical scaling study are conditional response probabilities, giving the likelihood that a particular response, $\mathbf{k}$, will be produced in the presence of a given stimulus, $j$. We shall denote this conditional probability by $p(k \mid j)$. The entire set of conditional response probabilities can be gathered into a matrix whose rows correspond to stimuli and whose columns correspond to responses, as in the table at the top of Figure 1. One result of applying a particular scaling model to this data matrix is a set of stimulus scale values, which we shall denote by $x_{j}$. where $j$ stands for a particular stimulus. and $j=1,2 \ldots, n$. The $x j$ 's give the value for each stimulus on the underlying sensory continuum.

\section{The Successive Intervals Model}

In the successive intervals model, $x_{j}$ is treated as a random variable, having a Gaussian distribution with

\section{Frequency Motrix}
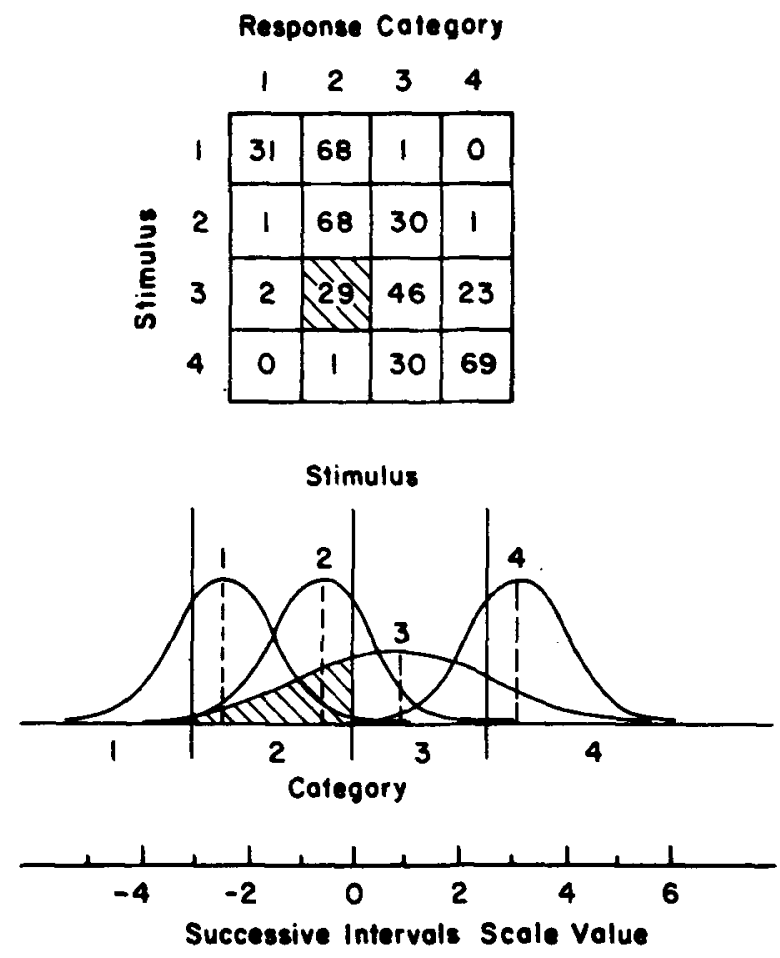

Figure 1. Idealized data lllustrating the successive intervals model. Frequencies in the stimulus-response matrix are generated by integrating the probabllity density corresponding to a stimulus over an interval corresponding to the particular response category.

mean $E\left(x_{j}\right)=\mu_{j}$ and standard deviation $o_{j}$. (The stimulus distributions are illustrated in Figure 1.) In addition, the sensory axis is partitioned into a series of successive, nonoverlapping intervals, each representing a particular judgment category, $k$, where $k=1$, $2, \ldots, \mathrm{m}$. It is assumed that the subject's categories are ordered along the sensory continuum, ${ }^{1}$ and that the successive intervals of the scaling model are ordered in the same way. The boundaries of the interval for response category $k$ are denoted by $x_{k-1}$ (the lower boundary) and $x_{k}$ (the upper boundary). The conditional probability that category $\mathrm{k}$ is chosen in response to stimulus $j$ is, in terms of the model, given by the area under the distribution for stimulus $j$ falling between the limits $x_{k-1}$ and $x_{k}$. Formally, this idea is expressed in the equation

$$
p(k \mid j)=\int_{x_{k}-1}^{x_{k}} \frac{1}{\sigma_{j} \sqrt{2 \pi}} \exp \left[\frac{-\left(x_{j}-\mu_{j}\right)^{2}}{2 \sigma_{j}^{2}}\right] .
$$

$x_{0}$, the lower boundary for category 1 , and $x_{m}$, the upper boundary for the topmost category $(m)$ are assumed to be $-\infty$ and $+\infty$, respectively. The distance between any two distributions on the sensory axis is 
given by the absolute value of the difference between the scale values, i.e., $\mathrm{d}_{\mathrm{ij}}=\left|\mu_{\mathrm{i}}-\mu_{\mathrm{j}}\right|$ gives the distance between stimulus $i$ and stimulus $j$. This distance function conforms to the Euclidean distance metric in one dimension. The distance, $\mathrm{d}_{\mathrm{ij}}$, is comparable to the $\mathrm{d}^{\prime}$ parameter in detection theory.

In the above discussion, it has been tacitly assumed that the boundary parameters are fixed on the sensory continuum. Torgerson (1958) has pointed out, however, that it is only necessary to assume that the $\mathrm{x}_{\mathrm{k}}$ 's are identically (normally) distributed, with $\mathrm{E}\left(\mathrm{x}_{\mathrm{k}}\right)$ $=\mu_{\mathrm{k}}$ and $\sigma_{\mathrm{k}}=\sigma$ (constant dispersions). When $\mathrm{x}_{\mathrm{k}}$ is assumed to be fixed, perhaps for purposes of computation, category boundary variance will inflate the stimulus dispersions by a constant amount equal to $\sigma^{2}$.

Least squares (Diederich, Messick, \& Tucker, 1957; Gulliksen, 1954; Torgerson, 1958), minimum normit (Bock, 1957), and maximum likelihood (Schönemann \& Tucker, 1967) solutions are available for the estimation of parameters. Since the resulting scale is an interval scale, a unit and origin must be defined. In the solution used here (maximum likelihood), the unit and origin are set so that the mean of the scale values is 0 and the average of the dispersions is 1 . Since the unit will incorporate variance due to category boundaries (assuming that the categories are in fact not fixed), the slope of the psychometric function will become smaller as variance in category boundaries is increased. This leads to the expectation that if an increase in the stimulus range contributes to unreliability in category placement on the sensory scale, this will result in a smaller slope for the psychophysical function than that obtained when the range of stimulus magnitudes is narrow. Put another way, the scale distance between two stimuli $\left(d_{i j}\right)$ will decrease as the range of stimulus magnitudes in the experiment is increased.

\section{The Unfolding Model}

In the unfolding model, each judgmental category is represented by a point on the sensory axis. This means that a distance can be defined between a stimulus and a category on the resulting psychophysical scale. Let $\mathrm{d}_{\mathrm{jk}}$ be the distance between stimulus $j$ and category $k$ on the underlying metric axis. Let $x_{j}$ again be the scale value of the stimulus $j$, and let $x_{k}$ be the value of the category $k$, where $k=1$, $2, \ldots, m$. Then $d_{j k}=\left|x_{j}-x_{k}\right|$. The $d_{j k}$ 's for different stimuli $(j=1,2, \ldots n)$ for a fixed category $k$ can be visualized if we think of the axis as folded at the point $\mathrm{x}_{\mathrm{k}}$. All stimuli can thus be made to lie on the positive side of the point $\mathrm{x}_{\mathrm{k}}$, and, if we set the origin at $x_{k}$, the resulting values (on the folded scale) for each stimulus will be just the distances $d_{j k}$. It is from this mental exercise that the scaling technique received its name (Coombs, 1964, Chap. 5).

In the formal model, the conditional probabilities $\mathrm{p}(\mathrm{k} \mid \mathrm{j})$ are treated as measures of the proximity between a stimulus and a category (cf. Carroll \& Wish, 1973). As proximity measures, the conditional probabilities are treated as nonmetric, in the sense that the $n \times m$ probabilities $p(k \mid j)$ serve merely to order the $\mathrm{n} \times \mathrm{m}$ proximities for the scaling analysis (Shepard, 1962a, b), rather than specifying their value directly. Thus, when $p(k \mid j)$ is large, the stimulus and category are assumed to be close to one another; when $p(k \mid i)$ is smaller than $p(k \mid j)$, stimulus $i$ is assumed to be more distant from category $k$ than is stimulus $j$. The scaling assumptions to follow now are those of Kruskal's (1964a, b) nonmetric multidimensional scaling procedure, and the unfolding model outlined here is simply a special case of this more general method. Let $d_{j k}$ be a best least-squares estimate of $d_{j k}$, the actual scale distance between stimulus $\mathrm{j}$ and category $\mathrm{k}$. $\mathrm{d}_{\mathrm{j} k}$ is assumed to be related to $p(k \mid j)$ by a monotonic decreasing function, which translates proximity measures $p(k \mid j)$ to distances. The scaling problem is solved by finding a set of values for $x_{j}$ and $x_{k}$ (and thus for the $d_{j k}$ 's) which minimize the quantity

$$
\sqrt{\frac{\sum_{j} \sum_{k}\left(d_{j k}-\dot{d}_{j k}\right)^{2}}{\sum_{j k} \sum_{k}\left(d_{j k}-\bar{d}\right)^{2}}},
$$

which is called the stress. ${ }^{2}$ (In this definition, $\overline{\mathrm{d}}$ is the average of all $d_{j k}$ 's.) The $d_{j k}$ 's are a function of the parameters to be estimated (the scale values), while the djk's are obtained from the empirical proximities [the $p(k \mid j)$ 's] by applying methods of least squares monotone regression. Kruskal's $(1964 a, b)$ multidimensional scaling program MDSCAL provides a computational solution to this problem. From the standpoint of the multidimensional scaling method, the present model is a restricted case in which (1) the dimensionality is assumed to be 1 , and (2) instead of the usual symmetric matrix of proximities, we have an $\mathrm{n} \times \mathrm{m}$ rectangular matrix of proximities relating objects in one set (the stimuli) to objects in a second set (the response categories). We shall see that these data provide sufficient constraint on the configuration of points for us to obtain a nondegenerate solution in one dimension. Since the unfolding model, in this realization, provides an interval scale, the origin is (arbitrarily) set so that the mean of the stimulus and category scale values is 0 . The unit is set so that their standard deviation is 1 . Also recovered in the scaling analysis is the proximity function relating conditional probabilities. $p(k \mid j)$ (or, alternatively, conditional response frequencies) to estimated distances, $\hat{d}_{\mathrm{jk}}$. For this model, manipulation of the range of stimulus magnitudes can be expected to alter the positions of the categories and the form of the proximity function, while leaving the stimulus scale values invariant. 
Two experiments were conducted to evaluate the extent of invariance of psychophysical scales obtained using each of these judgment models when stimulus range was manipulated. A conventional magnitude estimation study was also carried out to ensure that the manipulation of stimulus range was effective in altering the exponent of the psychophysical power function. In addition. a discrimination scale was constructed from subjects' magnitude estimates using the successive intervals method, in an effort to investigate whether invariance in the form of psychophysical equation can be attributed to the form of judgment employed by the subject.

\section{METHOD}

\section{Experiment 1: Visual Stimuli}

Subjects. Forty undergraduate and graduate students at Brandeis University were hired for the experiment.

Procedure. Groups of 10 subjects were assigned to the four experimental conditions, which included two stimulus ranges (wide and narrow) and two methods of judgment (quantitative and categorical). The stimuli were flashes of light lasting $500 \mathrm{msec}$, viewed with the right eye. The stimulus field subtended an angle of $12 \mathrm{deg} 15 \mathrm{~min}$. Luminance was controlled by means of neutral density filters placed in the image plane. Details of the Maxwellian viewing system and calibration procedures are described in Stecher. Sandberg. and Minsky (1970. ${ }^{3}$ Twenty stimulus intensities were included. For the wide-range condition, the stimulus luminance ranged from -.8 to $3.0 \log \mathrm{mL}$, in steps of $.2 \log \mathrm{mL}$. For the narrow-range condition, the stimuli ranged from -.8 to $1.1 \log \mathrm{mL}$, in steps of $.1 \log \mathrm{mL}$. The wide range (and the associated step size) was twice the narrow range, expressed in logarithmic units. The subject was dark adapted for 15 min before the experiment began. Stimulus order was randomized. Each stimulus was judged 10 times by each subject, for a total of 200 judgments in the experimental series. Data from the last 180 trials were retained for analysis. Since the cumulative exposure to the stimulus flashes was only $100 \mathrm{sec}$ spread over an experimental session lasting approximately $1 \mathrm{~h}$. differential light adaptation under the two conditions was presumably negligible.

Under the categorical judgment condition, subjects were required to rate the brightness of the stimulus on a 7 -point scale, ranging from low (1) to high (7) perceived magnitude. The only restriction in the use of the scale was that the subjects were asked to use all of the categories at some point in the experimental series. Under the quantitative judgment condition, subjects were asked to assign a number (magnitude estimate) to each stimulus representing the perceived stimulus magnitude. They were instructed that the number assigned was to be proportional to the magnitude of the stimulus, and examples of this principle were provided. No modulus was specified in the experiment.

\section{Experiment 2: Auditory Stimuli}

Subjects. Forty additional undergraduate and graduate students at Brandeis University were hired for this experiment.

Procedure. Again, four groups of 10 subjects were tested, representing two stimulus ranges and two methods of judgment. The stimuli were 1 -sec bursts of white noise produced by a Grason-Stadler Model 455-C noise generator, together with a Hewlett-Packard Model 350-C attenuator, and gated through a Grason-Stadler Model 829-E electronic switch, set for a rise/fall time of $5 \mathrm{msec}$. Stimulus levels were measured using a General Radio Model 1565-A sound-level meter (A weighting characteristic), together with a Model 1560-P83 earphone coupler. For the wide stimulus range condition, stimuli ranged in intensity from 42 to $80 \mathrm{~dB}$ re .0002 microbar, in steps of $2 \mathrm{~dB}$. For the narrow-range condition, stimuli ranged from 42 to $61 \mathrm{~dB}$ re

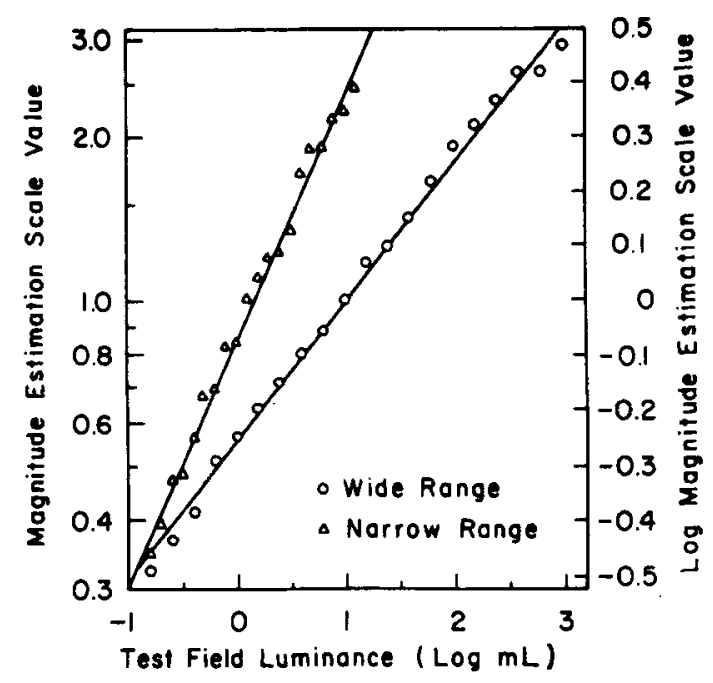

Figure 2. Psychophysical functions obtained for visual stimuli using the method of magnitude estimation. The slopes for the wide and narrow stimulus ranges are .256 and .446 , respectively.

.0002 microbar, in steps of $1 \mathrm{~dB}$. Again, the wide range of stimulus magnitudes was twice the narrow range, expressed in logarithmic units. Subjects were seated in a noise-attenuating chamber, and the stimuli were presented binaurally through Grason-Stadler TDH-39 headphones. The ambient noise within the testing chamber was below measurable level. Stimulus order was randomized. There were 200 observations per subject ( 10 per stimulus), and the first 20 trials were discarded as before. Groups of subjects were tested using the categorical and quantitative modes of judgment; the judgmental conditions were similar to those described for the first experiment.

\section{RESULTS}

\section{Magnitude Estimation}

Scale values obtained using the method of magnitude estimation were found to be influenced by the range of stimulus magnitudes for both sensory modalities. In each case, significant differences in exponents of fitted power functions were obtained for the wide- and narrow-range conditions. Data for the visual study are given in Figure 2. Shown are the arithmetic means of 90 responses to each of the 20 stimuli, plotted in double logarithmic coordinates. Individual subjects' responses were rescaled prior to averaging, so that the $1.0-\mathrm{mL}$ stimulus (for the wide range) or the $.1-\mathrm{mL}$ stimulus (for the narrow range) always had a value of 1 . The slopes of the two regression lines are .256 and .446 for the wide- and narrow-range conditions, respectively, and this difference in slopes is highly significant $[F(1,36)=$ $343.0, \mathrm{p}<.01$ ]. It should be pointed out that when a strictly linear model is compared with a model which includes quadratic terms, the slight curvilinearity exhibited for stimuli at the high end of each scale is statistically significant $[\mathrm{F}(2.34)=12.93, \mathrm{p}<.01]$. Nevertheless, in these and subsequent analyses, curvilinearity introduced by end points, while 


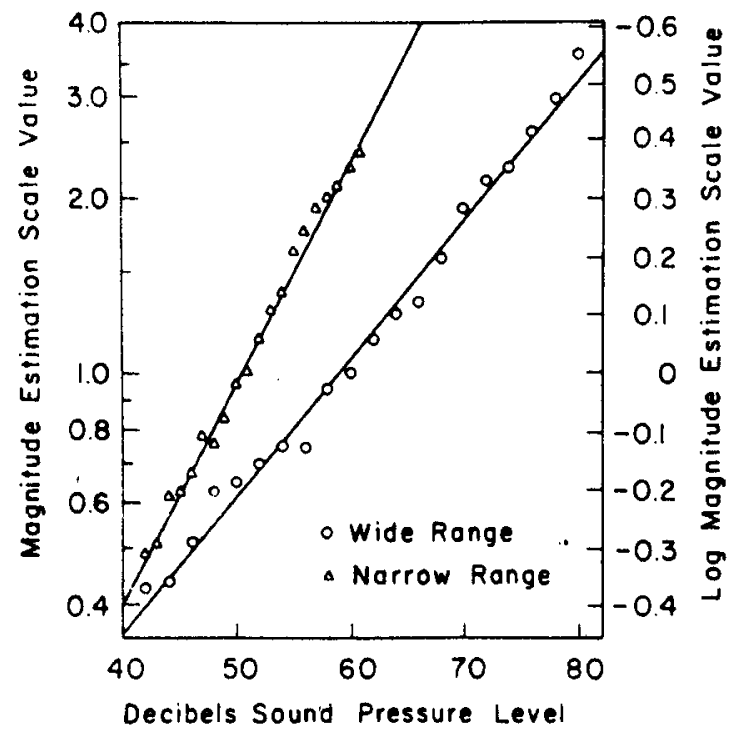

Figure 3. Psychophysical functions obtained for auditory stimuli using the method of magnitude estimation. The slopes for the wide and narrow stimulus ranges (divided by 10 ) are .236 and .381 .

reported, will be generally disregarded in fitting theoretical functions to the data.

Magnitude estimation scales obtained in the auditory experiment are shown in Figure 3; again, the slopes differ $[F(1,36)=173.68, p<.01]$, with the steeper slope again being that for the narrow range condition (.381, compared with .236 for the wide-range condition). ${ }^{4}$ Again, significant curvilinearity was present in the psychophysical functions $[F(2,34)=6.54, \mathrm{p}<.01]$.

\section{Successive Intervals}

Maximum likelihood estimates of the successive intervals model parameters were next computed using an iterative procedure due to Schönemann and Tucker (1967). The data for the analysis were the matrices of conditional response frequencies, which were tallied directly from the subjects' categorical responses. Separate analyses were carried out for each range of stimulus magnitudes, and for each sensory modality. As an index of fit, chi-square values ranged from 73.1 to 130.4 , with $76 \mathrm{df}$, and the average of absolute deviations of fitted from observed conditional response probabilities ranged from .018 to .022. Sinilar sets of successive intervals scale values were then computed for the quantitative judgment data. To facilitate this analysis, subjects' magnitude estimates were grouped into seven categories. (This was accomplished for each subject by dividing the range of magnitude estimates into $1 / 6 \mathrm{~s}$ and then dividing the first $1 / 6$ into halves.) This procedure can be likened to the grouping of data commonly employed in constructing frequency histograms. Analyses of the resulting derived matrices of conditional response frequencies led to successive interials scale values lor the wide- and narrow-range conditions. which could be compared with those obtained using the categorical judgment procedure. Chi-square values obtained in fitting data obtained from quantitative judgments ranged from 62.4 to 133.9, with $76 \mathrm{df}$; the average of absolute deviations of titted from observed conditional probabilities here ranged from .017 to .023 .

Scale values obtained in the visual experiment for both quantitative and categorical judgment are shown in Figure 4. For categorical judgment, scale values for both stimulus ranges appear to be approximately linearly related to log stimulus magnitude. Moreover, successive intervals scale values appear to be invariant to a reasonable approximation as the type of judgment (categorical or quantitative) is altered. A linear model in which jud gment variables were deleted was nearly as good in fitting the data of Figure 4 as was a model which included such variables $[\mathrm{F}(4,72)=$ 1.38]. There was a clear difference in slope for the two stimulus ranges [2.31 for the narrow range and 1.85 for the wide range, with $F(1,72)=34.16, p<.01$ ], indicating that there is an increase in judgmental variability when the subject attempts to partition a wide stimulus range into a fixed set of successive intervals. Again, there was some evidence of curvilinearity in the psychophysical functions, with $\mathrm{F}[4,68)=14.69, \mathrm{p}<.01$.

Scale values obtained in the auditory study are shown in Figure 5. Here again, successive intervals scale values appear to be approximately linearly related to $\log$ stimulus magnitude, regardless of the range of magnitudes or the type of judgment

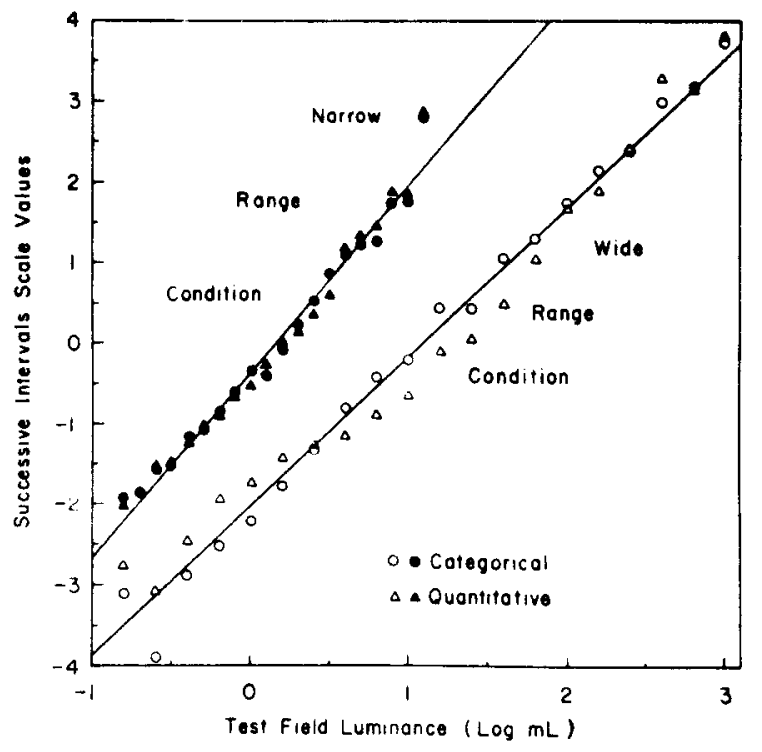

Figure 4. Psychophysical functions obtained by applying the successive intervals model to visual data obtained using categorical and quantitative modes of judgment. 


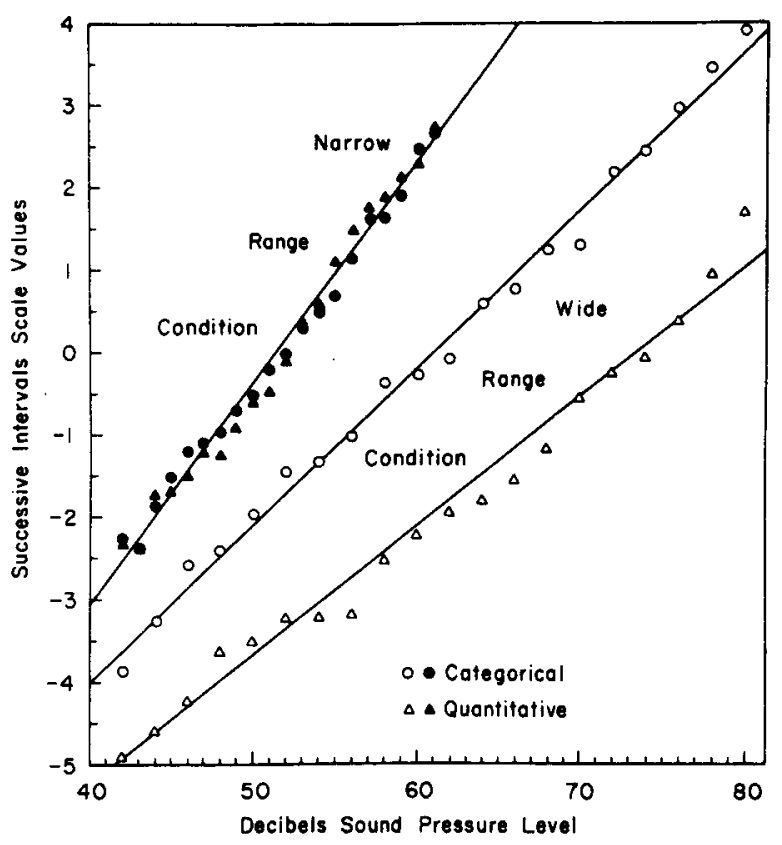

Figure 5. Psychophysical functions obtained by applying the successive intervals model to auditory data obtained using categorical and quantitative modes of judgment. The data for the wide-range, quantitative judgment condition have been displaced downward two units.

employed. However, in this case, a significant interaction between stimulus magnitude, stimulus range, and type of judgment $[\mathrm{F}(2,72)=16.43$, $\mathrm{p}<.01]$ indicates that, in addition to there being greater judgmental variability in partitioning a wide range of stimulus magnitudes, subjects under these conditions show greater variability when making quantitative judgments than when making categorical judgments. This is seen in the smaller slope for quantitative judgment than that for categorical judgment, when the stimuli covered a wide range of magnitudes. There was again some evidence of curvilinearity in the data, with $F(4,68)=11.27$, $\mathrm{p}<.01$.

The stimulus dispersions obtained in all of the successive intervals analyses are shown in Figure 6. At the top are plotted the dispersions for the first experiment; those for the second experiment are at the bottom. The fitted lines represent the average of the dispersions for a particular experimental condition, and this average is always (by construction) 1. While unequal stimulus dispersions are tolerated under the successive intervals model, there is no evidence of systematic departures from an assumption of constant dispersions over the range of stimulus magnitudes employed. The successive intervals scales can therefore be described here as equidiscriminability scales.

\section{The Unfolding Analysis}

The matrices of conditional response frequencies obtained from categorical judgments were treated as matrices of proximity measures for the purposes of the unfolding analysis. Four such analyses were carried out, with separate analyses for the two sense modalities and the two stimulus ranges. To demonstrate the uniqueness of the resulting scale values, each of these analyses was then repeated using a second, different starting configuration. The first starting configuration used was a linear function of $\log$ stimulus magnitude, and the second was an arbitrary monotonic (cubic) transformation of the first (linear) starting values. The solutions obtained using the two starting configurations were closely similar; the average absolute discrepancy in scale values obtained in the two analyses was .022 . The data reported here were obtained using the first starting configuration, and are shown in Figure 7 for both the visual and auditory experiments. Stress values for the wide and narrow conditions were .088 and .188 , respectively, for the visual data, and .131 and .212 for

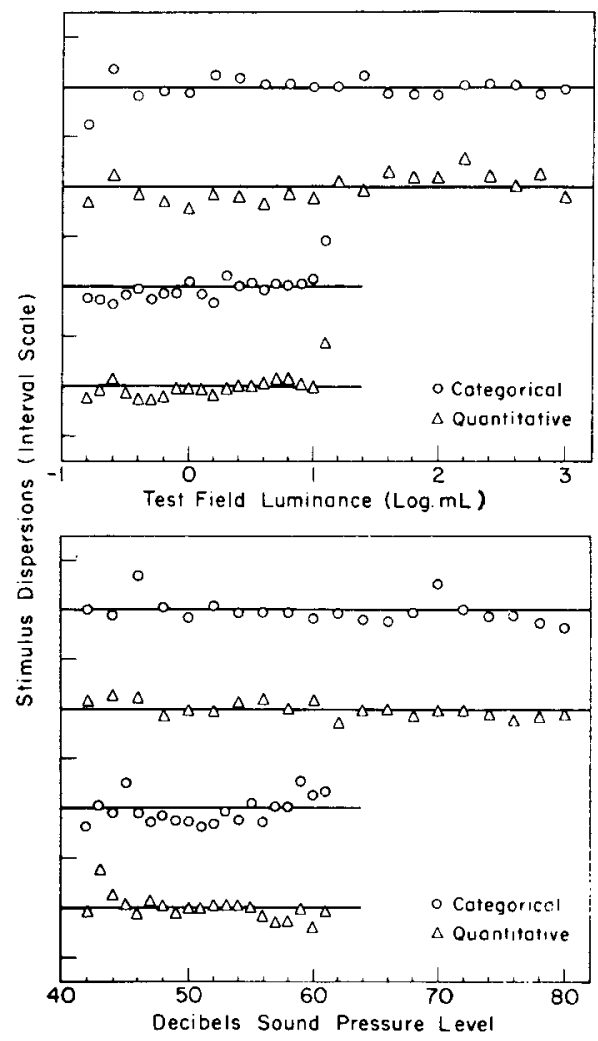

Figure 6. Stimulus dispersions obtained in the successive intervals analyses, for visual (top panel) and auditory (bottom panel) stimuli. In each panel, the wide stimulus range conditions are presented at the top and the narrow range conditions at the bottom. For each condition, the fitted line represents the mean of the dispersions, which is in all cases 1 . The unit on the ordinate is equal to one dispersion. 


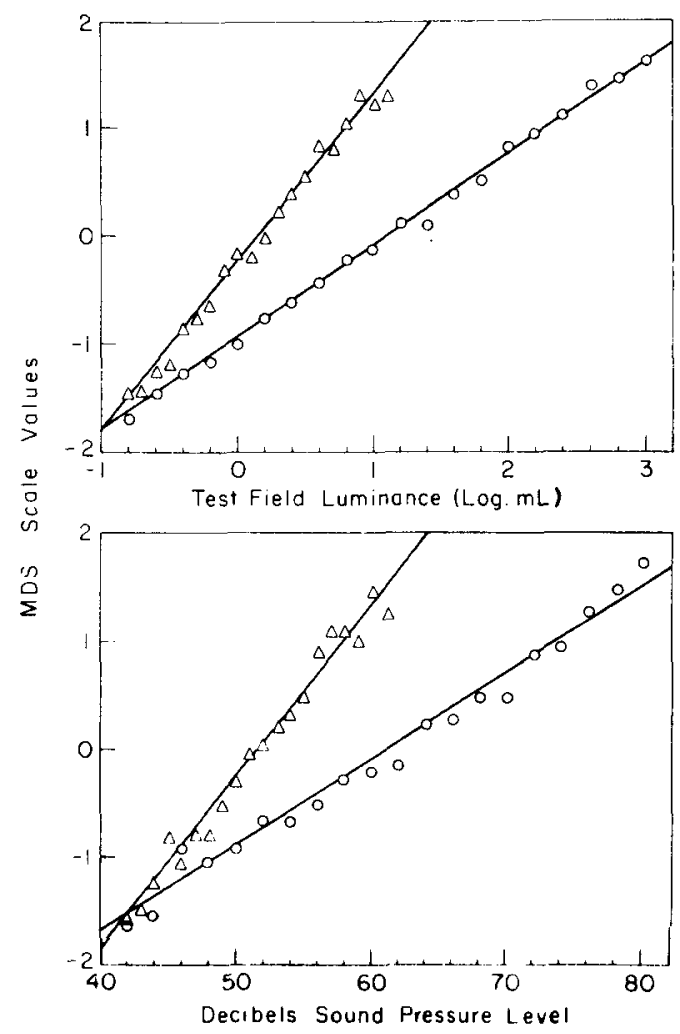

Figure 7: Psychophysical functions obtained for visual (top panel) and auditory (bottom panel) stimuli based upon the unfolding model. The data are obtained from categorical judgments. Each function is scaled so that the mean of the scale values associated with stimuli and response categories is 0 , and the standard deviation is 1 . The steeper function is, in each case, that obtained for the narrow range.

the auditory data. The psychophysical scale values are in all cases logarithmic functions of stimulus magnitude; the linearity in the semi-log plots of Figure 7 is reflected in correlations of .998 and .995 for the wide and narrow stimulus ranges in the visual experiment, and similar correlations of .991 and .990 for the auditory experiment.

While the slopes of psychophysical functions obtained under the successive intervals model could be meaningfully compared (since the units are determined there by the stimulus dispersions and category boundary variance), the same is not true for the scale values obtained under the unfolding model. Here, the slopes have been arbitrarily adjusted so that the variance of the scale values (including those for the categories) is 1. However, metric information about the ability of a subject to discriminate among stimuli is contained in the proximity functions which are obtained as a result of the scaling procedure. These functions are shown in Figure 8 (solid lines). The ordinate represents the empirical measure of proximity (the conditional response frequency), and the abscissa represents the estimated scale distance. $\mathrm{d}_{\mathrm{jk}}$. In both the visual and auditory experiments, the estimated scale distance grows more rapidly with decreases in response frequency when the stimulus range is narrow than when it is wide. The form of the proximity function otherwise appears to be similar for the two stimulus ranges. If we correlated $\hat{d}_{\mathrm{jk}}$ values for the wide and narrow ranges for a set of comparable response frequencies, we find $r=.981$ for the visual experiment and $r=.980$ for the auditory experiment. The corresponding regression equations for predicting narrow-range distances from those for the wide range are: $\mathrm{y}=1.261 \mathrm{x}-.026$, for the visual experiment, and $y=1.263 x-.096$, for the auditory experiment. Since the intercepts are both nearly zero, these equations indicate that an expansion of the scale unit for the wide-range condition will be sufficient to equate the psychophysical scales with respect to the underlying proximity function. If this is done, the slopes of the psychophysical functions, plotted against log stimulus magnitude, become: 1.102 and 1.590 , for wide and narrow stimulus ranges in the visual experiment, and .104 and .163 , respectively, for the auditory experiment. These results show that the scale difference (discriminability) between two stimuli increases when the range of stimulus magnitudes is reduced.

\section{DISCUSSION}

Both judgment models provide psychophysical

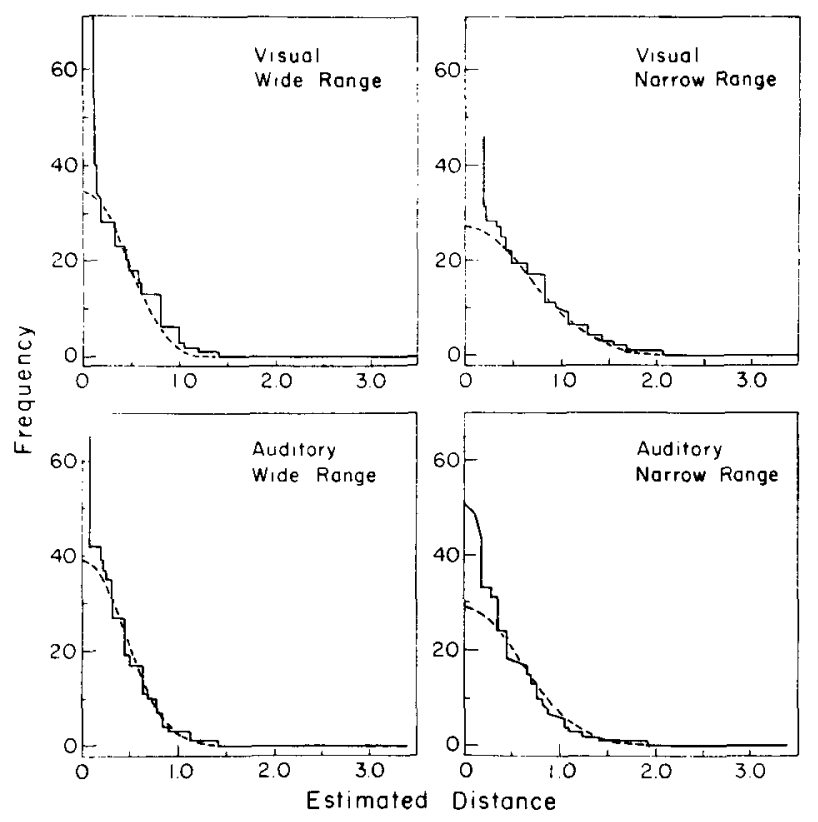

Figure 8. Distance functions obtained in fitting the unfolding model, using the MDSCAL program. The dashed lines represent approximations obtained from the successive intervals analysis; see text for an explanation. 
Table 1

Parameters Reflecting Judgment Categories for Successive Intervals and Unfolding Models*

\begin{tabular}{|c|c|c|c|c|c|c|c|c|c|}
\hline \multirow[b]{2}{*}{ Category } & \multirow{2}{*}{$\begin{array}{c}\text { Category } \\
\text { Boundary }\end{array}$} & \multicolumn{4}{|c|}{ Visual Stimuli } & \multicolumn{4}{|c|}{ Auditory Stimuli } \\
\hline & & Wide & Range & Narrow & Range & Wide & Range & Narrow & Range \\
\hline 1 & & -1.43 & & -1.63 & & -1.49 & & -1.60 & \\
\hline 2 & $1-2$ & -.98 & -1.28 & -1.02 & -1.48 & -1.02 & -1.32 & -.94 & -1.35 \\
\hline 3 & $2-3$ & -.33 & -.68 & -.60 & -.89 & -.45 & -.62 & -.41 & -.68 \\
\hline 4 & $3-4$ & .16 & -.11 & .05 & -.29 & .18 & -.10 & .26 & -.15 \\
\hline & $4-5$ & & .37 & & .33 & & .41 & & .32 \\
\hline 5 & $5-6$ & .56 & .82 & .88 & .89 & .68 & .96 & .69 & .80 \\
\hline 6 & $6-7$ & $\begin{array}{r}.99 \\
.53\end{array}$ & 1.29 & $\begin{array}{l}1.37 \\
1.80\end{array}$ & 1.62 & 1.14 & 1.53 & 1.27 & 1.57 \\
\hline 7 & & 1.53 & & 1.80 & & 1.79 & & 1.77 & \\
\hline
\end{tabular}

*Values are presented in the metric of the unfolding analysis.

scales which are logarithmic functions of stimulus magnitude. The scale values also proved to be linearly invariant with changes in the range of stimulus magnitudes employed in the experiment. However, in each case, the measure of discriminability between the two stimuli $\left(\mathrm{d}_{\mathrm{ij}}\right)$ changed with changes in the stimulus range, so that discriminability decreased as stimulus range was increased. This common set of findings, resulting from the application of two scaling methods, suggests that the two models may represent simply alternate ways of representing the same underlying judgmental process. If this is true, then the question arises: Can an alternate set of parameters be found for the successive intervals model which will resemble those of the unfolding model? More specifically, if we substitute a set of point scale values for the category intervals of the successive intervals model, can we then devise a proximity function which will resemble the function recovered in the unfolding analysis?

Such a modification of the successive intervals model can be constructed. We begin by making two simplifying assumptions: (1) that the dispersions in the successive intervals model are constant, and (2) that the category intervals all have the same width. Data relevant to this second assumption are presented in Table 1, which gives the category boundary parameters for the successive intervals analysis. (Note that the metric here is that of the unfolding analysis.) In each analysis, the category intervals are roughly of equal widths; for example, values for the visual experiment are $.60, .57, .48, .45$, and .47 , for the wide-range condition, and $.59, .60, .62, .56$, and .73 , for the narrow-range condition. A further point to notice in Table 1 is that the scale values for the categories obtained in the unfolding analysis fall, in every case, within the corresponding category intervals obtained in the successive intervals analysis. For example, in the case of the visual experiment, the value -1.43 obtained for category 1 under the wide-range condition falls within the first of the successive intervals, which is bounded at the bottom at -1.28 ; the value for the second category $(-.98)$ falls within the interval -1.28 to -.68 ; and so forth. It should further be noted that the category values, by and large, fall at the midpoints of the corresponding category intervals, and it is this approximation that leads to the definition of a category scale value. Let $\mathrm{x}_{\mathbf{k}}$ ' be the midpoint of the $\mathrm{k}^{\text {th }}$ category interval in the successive intervals analysis; $\mathbf{x}_{\mathbf{k}}{ }^{\prime}$ is defined to be the scale point corresponding to category $k$. The distance between category $k$ and any stimulus (j) can then be defined to be $d_{j k}=\left|\mu_{j}-x_{k}{ }^{\prime}\right|$, where $\mu_{j}$ is the stimulus scale value in the successive intervals analysis. The proximity function relating $d_{j k}$ to the conditional response probability can be generated by simply calculating the area under the stimulus distribution (which has unit variance) within the interval $x_{k}{ }^{\prime}-d$ to $x_{k}{ }^{\prime}+d$ (where $d$ is half the category interval width) for different values of $\mu_{j}$ (and, therefore, of $\mathrm{d}_{\mathrm{jk}}$ ). These constructed proximity functions are plotted as the dashed lines in Figure 8. It can be seen that the fit to the proximity functions obtained under the unfolding model is close, except for a few of the largest frequency values. This is to be expected in view of our failure to recognize, when we constructed the proximity function, that the top and bottom successive intervals are in fact unbounded. Stimuli located below the bottom category boundary or above the topmost category boundary will be judged in the corresponding category with higher frequencies than those we have predicted under our simplifying assumptions.

Our conclusion, then, is that the proximity functions generated from the successive intervals model by letting category interval midpoints serve as the category parameters are similar in form to the proximity functions recovered in the unfolding analysis. Put the other way, the recovered proximity 
functions are consistent with the normality assumptions of the successive intervals model. The two judgment models can be thought of as alternate mathematical representations for the same judgmental process. This does not mean, however, that the models are interchangeable in any field of application. While the unfolding model is less restrictive in its assumption of a nominal rather than ordinal scale of judgment and in its weak monotonicity restriction on the proximity function, it is stronger than the successive intervals model in assuming that there is a common proximity function which applies to all stimuli. This latter restriction is equivalent to assuming, within the successive intervals model, that there are constant stimulus dispersions.

The representation of judgmental categories as points on the underlying psychophysical scale suggests an interesting way of viewing the process of psychophysical judgment, which might be termed a theory of idealized stimuli. Suppose that the subject establishes, in the course of a scaling experiment, a series of idealized reference stimuli corresponding to each of the judgmental categories he is asked (or chooses) to use. Suppose further that he judges stimulus magnitudes by selecting the category whose momentary value is closest to the value of the stimulus impression. Judgmental variability will be due to an imperfect memory for the position of the idealized stimulus corresponding to each of the judgmental categories (cf. Durlach \& Braida, 1969). Increasing the range of stimulus magnitudes to be represented by a fixed number of categories will decrease the precision with which the idealized stimulus is represented in memory (Gravetter \& Lockhead, 1973).

\section{Judgmental Models for Magnitude Estimation}

When successive intervals scales were constructed from subjects' quantitative estimates of sensory magnitude, the resulting scales were again logarithmic functions of stimulus magnitude and were linearly related to discrimination scales based upon the successive intervals and unfolding models. The type of judgment required of the subject appears to be unimportant; discrimination scales built from magnitude estimation judgments do not differ in any important way from those built from categorical judgments. Moreover, the invariance of the underlying discrimination scale under conditions where the magnitude estimation scale is curvilinearly transformed (by varying stimulus range) suggests that the discrimination scale may represent a more basic (sensory) process, and that the judgment of magnitude represents a further processing of underlying sensory information (Treisman, 1964).

It is easy to show, for example, that a change in the unit of the discrimination scale due to manipulation of stimulus range produces a linear transformation of the logarithm of the magnitude estimation scale (and a nonlinear transformation of the untransformed magnitude estimation scale) when the discrimination (category) scale is related to the magnitude estimation scale by a logarithmic function (Helm, Messick, \& Tucker, 1961),

$$
C_{i j}=a \log M_{i j}+b,
$$

where $C_{i j}$ is the category (discrimination) scale value for stimulus $i$ under condition (stimulus range) $j(j=$ $1,2), M_{i j}$ is the corresponding magnitude estimation scale value, and $a$ and $b$ are constants which are independent of stimulus range. A change in the unit of the discrimination scale due to manipulation of stimulus range implies that

$$
\mathrm{C}_{\mathrm{i} 2}=\mathrm{d} \mathrm{C}_{\mathrm{i} 1}
$$

where $\mathrm{d}$ is a constant. Substituting Equation 1 into Equation 2 yields the relation:

$$
\log \mathrm{M}_{\mathrm{i} 2}=\mathrm{d} \log \mathrm{M}_{\mathrm{i} 1}+\mathrm{b}(\mathrm{d}-1) / \mathrm{a} .
$$

Since $\log \mathrm{M}_{\mathrm{ij}}$ is a linear function of $\log$ stimulus magnitude (i.e., conforms to a power law), this implies that a change in precision of judgment also produces a change in power law exponent and that the ratio of slopes of the psychometric functions for different stimulus ranges should be the same for discrimination scales and log magnitude estimation scales. For instance, in the present study, the visual data yielded slope ratios (narrow range to wide range) of 1.32 for the discrimination scale and 1.74 for the log magnitude estimation scale; the corresponding values for the auditory data were 1.75 and 1.61. The variability in power law exponents means that ratios of magnitude estimation values will not be preserved under changes in stimulus range, since this ratio is determined by the value of the exponent. However, ratios of differences in log magnitude estimation scale values will be preserved. Let $M_{i}=k S_{i} m$ be the power function relating magnitude estimation scale value $\left(\mathrm{M}_{\mathrm{i}}\right)$ to stimulus magnitude $\left(\mathrm{S}_{\mathrm{i}}\right)$ for a fixed set of conditions, so that $\mathrm{k}$ and $\mathrm{m}$ are constants. Then, for three stimuli, $S_{1}, S_{2}$, and $S_{3}$,

$$
\log \mathrm{M}_{\mathrm{i}}=\log \mathrm{k}+\mathrm{m} \log \mathrm{S}_{\mathrm{i}}
$$

so that the ratio of differences becomes

$\frac{\log M_{3}-\log M_{1}}{\log M_{2}-\log M_{1}}$

$$
=\frac{\left(\log k+m \log S_{3}\right)-\left(\log k+m \log S_{1}\right)}{\left(\log k+m \log S_{2}\right)-\left(\log k+m \log S_{1}\right)}
$$




$$
=\frac{\log S_{3}-\log S_{1}}{\log S_{2}-\log S_{1}}
$$

Since this ratio does not depend upon the value of the exponent $\mathrm{m}$. it is invariant with changes in stimulus range. The log magnitude estimation scale is thus an interval scale, and can be expected to behave in the same manner as the discrimination scale. In particular, the slope $m$ in Equation 4 will increase by a factor $\mathrm{d}$ (Equation 3) as precision of judgment improves, and this will be the same factor by which the unit of the discrimination scale is also changed (Equation 2). Since the $\log$ magnitude estimation wale and discrimination scale behave in a similar fashion with respect to the effects of stimulus range, there would seem to be little basis for preferring one scaling technique over the other. However, a study of information processing models for psychophysical judgment in direct magnitude estimation leads to the conclusion that models in which judgments are assumed to be based upon ratios of sensory magnitudes will not account for the effects of stimulus range without considerable elaboration, while a model which is similar to the judgment model suggested above for categorical judgment can account for the observed effects of stimulus range.

In producing magnitude estimation judgments, the observer is assumed to make a direct comparison of the ratio of a sensory magnitude associated with a stimulus to that of a modulus, which is assigned the scale value 1 (Stevens, 1957). Since the modulus is not present at the moment of judgment, it must be assumed to be an impression of stimulus magnitude which is retained in memory. Let $M_{\bmod }$ be the momentary value of the modulus on the underlying sensory scale; $\mathbf{M}_{\text {mod }}$ will be a random variable, whose expected value and variance may depend, among other things, on the current value of the stimulus and on the range of stimuli employed in the experiment. Since the magnitude estimation scale value is obtained by averaging estimates obtained over a series of replications, the scale value assigned the stimulus is given by the expected value of a ratio:

$$
E\left(M_{i}\right)=E\left(k S_{i}{ }^{m} / M_{\text {mod }}\right)
$$

in which the numerator represents the value of the stimulus, and the denominator represents the value of the modulus. Taking logarithms, we can write

$$
E\left(\log M_{i}\right)=\log k+m \log S_{i}-E\left(\log M_{\text {mod }}\right)
$$

We shall now consider a number of ways in which the value of the modulus might be related to particular experimental conditions.

(a) The variance of the modulus depends upon the range of stimulus magnitudes employed, but the modulus is unbiased, i.e., $\mathrm{E}\left(\log \mathbf{M}_{\bmod }\right)=0$. For this model, no stimulus range effects are predicted.

(b) The expected value of $\log \mathbf{M}_{\text {mod }}$ increases with increases in the stimulus range or, perhaps, with $\operatorname{Max}\left(S_{\mathfrak{i}}\right)$, but does not depend upon the value of the current stimulus. For this model, the stimulus range effect is confined to the intercept in the plot of $\log \mathrm{M}_{\mathrm{i}}$ against $\log \mathbf{S}_{\mathbf{i}}$.

(c) The momentary value of the modulus is biased in the direction of the current stimulus value. Then large stimulus values will increase the value of $M_{\bmod }$, leading to an underestimate of their scale values, and the reverse will be true for stimuli having small values. For example, if $E\left(\log M_{\text {mod }}\right)=\log p+q \log S_{i}$, the log magnitude estimation scale values are given by

$$
E\left(\log M_{i}\right)=\log k-\log p+(m-q) \log S_{i} .
$$

Bias in placing the modulus on a given trial leads to a change in both the exponent and scale factor in the power law. However, here the bias is unrelated to the stimulus range.

(d) Suppose, finally, that the modulus depends both on the current stimulus value and on the maximum stimulus value employed in the experiment, so that the amount of bias due to the current stimulus depends upon the stimulus range (or $\left.S_{\max }\right)$. Specifically, if we assume that the $\mathrm{E}(\log$ $\mathbf{M}_{\text {mod }}$ ) is a linear function of the logarithm of stimulus intensity and that the coefficient of $\log S_{i}$ is determined by the stimulus range, given by the function $q\left(S_{\max }\right)$, then

$$
E\left(\log M_{i}\right)=\log k-\log p+\left[m-q\left(S_{\max }\right)\right] \log S_{i} \cdot(9)
$$

Here there is an effect of stimulus range on the exponent of the power function, as demanded by the data. Thus, in order to account for the effects of stimulus range, it must be assumed that the momentary value of $\log \mathrm{M}_{\bmod }$ is hiased by an amount which is a function of the intensity of the current stimulus, and that the amount of this bias (the coefficient of $\log S_{i}$ in the biasing function) depends upon the range of stimuli employed. However, Equations 8 and 9 suggest that magnitude estimation scale values will always be determined to some measure by judgmental bias due to errors in locating the modulus on the judgmental scale.

An alternative model for magnitude estimation judgment is based upon the previous analysis of categorical judgment. If subjects are not judging ratios of stimulus magnitudes to the remembered value of a single modulus, then there remains the possibility that they may be assigning quantitative judgments on the basis of perceived similarity of the stimulus to a set of remembered sensory values which correspond to the categories used in making quantitative judgments. Under this proposal, we would expect discrimination scales (e.g., successive 
intervals scales) based upon an analysis of precision in assigning magnitude estimates to stimuli to be similar in form to discrimination scales based upon categorical judgment, and this of course was the case. The equivalence of these discrimination scales suggests that the principles involved in quantitative judgment of perceived stimulus magnitude can be regarded as similar to those employed in other types of psychophysical judgment. In the case where instructions require that the observer make quantitative estimates of ratios of perceived magnitudes, the magnitude estimates chosen are exponentially related to sensory magnitudes (from Equation 1), as represented by the discrimination scale. Note that the form of this function is independent of stimulus range. According to this model, the effect of stimulus range on the power law exponent is entirely due to the change in precision of judgment and its effect on the unit of the discrimination scale, which has been empirically determined.

\section{REFERENCES}

BAIRD, J. C. A cognitive theory of psychophysics. II. Fechner's liw and Sievens' law. Scandinavian Journal of Psychology, $1970,11,89.102$.

Bock, R. D. Note on the least squares solution for the method of successive categories. Psychometrika, 1957, 22, 231-240.

Bralda, L. D., \& Durlach, N. I. Intensity perception. Il. Resolution in one-interval paradigms. Journal of the Acoustical Sicciety of America, 1972, 51, 483-502.

Carroll, J. D., \& Wish, M. Multidimensional perceptual models and measurement methods. In E. C. Carterette and M. P. Friedman (Eds.), Handbook of perception. New York: Academic Press. 1973.

Coоmвs, C. H. A theory of data. New York: Wiley, 1964.

Diederich, G. W., Messick, S. J., \& Tucker, L. R. A general least squares solution for successive intervals. Psychometrika, 1957, 22, 159-173.

Durlach, N. I., \& Braida, L. D. Intensity perception. I. Preliminary theory of intensity resolution. Journal of the Acoustical Society of America, 1969, 46, 372-383.

EISLER, H. On the problem of category scales in psychophysics. Scandinavian Journal of Psychology, 1962, 3, 81-87.

Galanter, E. H., \& Messick, S. J. The relation between category and magnitude scales of loudness. Psychological Review, 1961, 68, 363-372.

Gravetter, F., \& Lockhead, G. D. Criterial range as a frame of reference for stimulus judgment. Psychological Review, 1973 , 80, 203-216.

Green, D. M., \& Swets, J. A. Signal detection theory and psychophysics. New York: Wiley, 1966.

Gulliksen, H. A least squares solution for successive intervals assuming unequal standard deviations. Psychometrika, 1954, 19. $117-140$.

HaRTMAN, E. B. The influence of practice and pitch-distance between tones on the absolute identification of pitch. American Journal of Psychology, 1954, 67, 1-14.

Heim, C. E., Messick. S., \& Tucker. L. R. Psychological models firr relating discrimination and magnitude estimation seales. Parchological Review, 1961, 68, 167-177.

KRUSKal, J. B. Multidimensional scaling by optimizing goodness of tit to a nonmetric hypothesis. Psychometrika, 1964, 29, 1-27. (a)

KRUSKal, J. B. Nonmetric multidmensional scaling: A numerical method. Psychomerika, 1964, 29, 115-129. (b)

LuCE, R. D., \& Mo, S. S. Magnitude estimation of heaviness and loudness by individual subjects: A test of a probabilistic response theory. British Journal of Mathematical and Statistical Psychology, 1965, 18, 159-174.

Pollack, I. The information of elementary auditory displays. II. Journal of the Acoustical Society of America, 1953, 25, 765-769.

Poulton, E. C. The new psychophysics: Six models for magnitude estimation. Psychological Bulletin, 1968, 69, 1-19.

SAFFIR, M. A. A comparative study of scales constructed by three psychophysical methods. Psychometrika, 1937, 2, 179-199.

Schönemann, P. H., \& Tucker, L. R. A maximum likelihood solution for the method of successive intervals allowing for unequal stimulus dispersions. Psychometrika, 1967, 32, 40,3-417.

ShEPARD, R. N. Analysis of proximities: Multidimensional scaling with an unknown distance function. I. Psychometrika. $1962,27,125-140$. (a)

ShEPARD, R. N. Analysis of proximities: Multidimensional scaling with an unknown distance function. II. Psychometrika, 1962, 27, 219-246. (b)

Stecher, S., Sandberg, B., \& Minsky, P. J. Successive luminance difference thresholds and brightness as a function of the interstimulus interval and durations of successive flashes. Perception \& Psychophysics, 1970, 7, 79-85.

Stevens, S. S. On the psychophysical law. Psychological Review, 1957, 64, 153-181.

Stevens, S. S. Ratio scales, partition scales, and confusion scales. In H. Gulliksen and S. Messick (Eds.), Psychological scaling: Theory and applications. New York: Wiley, 1960.

Stevens, S. S., \& Galanter, E. H. Ratio scales and category scales for a dozen perceptual continua. Journal of Experimental Psychology, 1957, 54, 377.411.

Teghtsoonian, R. On the exponents in Stevens' law and the constant in Ekman's law. Psychological Review, 1971, 78, 71-80.

TORGERSON. W. S. Theory and methods of scaling. New York: Wiley, 1958.

Treisman, M. Sensory scaling and the psychophysical law. Quarterly Joumal of Experimental Psychology, 1964, 16. 11-22.

\section{NOTES}

1. The subject's scale of judgment is thus assumed to be an ordinal scale in the model for successive intervals. In contrast, this judgment scale is assumed to be an interval scale in the construction of category-rating scales, and is assumed to be a ratio scale in the method of magnitude estimation (Stevens, 1957). In the unfolding model to be described, the judgments are assumed to be nonmetric, or simply nominal.

2. The stress formula used here is called STRESSFORM2 in Kruskal's program. MDSCAL.

3. The author wishes to thank Sidney Stecher for the use of the optical system.

4. The slopes are here divided by 10 , in order to make them comparable to values in the visual experiment, in which the abscissa represents a simple logarithmic scale rather than a decibel scale.

5. The author wishes to thank Peter Schönemann for furnishing a copy of his computer program for successive intervals scaling.

6. Midpoints are, of course, not defined for the top and bottom intervals, which are open-ended.

(Received for publication October 15, 1973; revision received April 29, 1974.) 